\title{
Task-set inhibition in chunked task sequences
}

\author{
DARRYL W. SCHNEIDER \\ Vanderbilt University, Nashville, Tennessee
}

\begin{abstract}
Exploring the hierarchical control relationship between different levels of representation and processing is important for understanding how the mind controls itself. In the present study, the relationship between chunking (a sequence-level process) and task-set inhibition (a task-level process) in the performance of task sequences was investigated to evaluate the hypothesis that within-chunk facilitation reduces the $n-2$ repetition cost (slower performance for $n-2$ task repetitions than for $n-2$ task switches) attributed to task-set inhibition. An experiment is reported in which subjects were induced to chunk sequences such that $n-2$ repetitions occurred within or between chunks. Direct evidence of chunking was obtained, and $n-2$ repetition cost was smaller when $n-2$ repetitions occurred within chunks than between chunks. These findings are consistent with an elaborated hypothesis that attributes the reduction in $n-2$ repetition cost to priming of task goals rather than direct modulation of task-set inhibition.
\end{abstract}

Understanding how the mind controls itself is a fundamental aim of cognitive psychology. Recent studies of executive control have been centered around task-switching situations in which people repeat or switch tasks in rapid succession. People are slower and more error prone at switching than repeating tasks, and this switch cost is often thought to reflect the functioning of myriad executive control processes, such as attention shifting, goal retrieval, response-set adjustment, and inhibition of prior task sets (Monsell, 2003). This last process - task-set inhibition-is considered to be a basic executive control process (Mayr $\&$ Keele, 2000) that functions at the task level to enable the performance of different task sequences.

An important issue that has received limited attention is how task-level processing - such as task-set inhibition - is affected by sequence-level processing that is associated with instantiating and maintaining a task sequence in memory. Recent research has provided evidence of a hierarchical control relationship between the sequence and task levels, such that what happens at one level is affected by what happens at the other level (Schneider \& Logan, 2006). The purpose of the present study was to explore this hierarchical control relationship by investigating how a task-level process (task-set inhibition) is affected by a sequence-level process (chunking) when switching tasks in sequences.

\section{From the Task Level to the Sequence Level}

When switching between three tasks (denoted A, B, and $\mathrm{C}$ ), people are slower at $n-2$ task repetitions (ABA) than $n-2$ task switches (CBA). The dominant interpretation of this $n-2$ repetition cost is task-set inhibition (Mayr $\&$ Keele, 2000): When performing an ABA sequence, switching from task A to task B is thought to involve in- hibition of the task set for task A; switching from task B back to task A requires time to overcome this inhibition, resulting in a performance cost. When performing a CBA sequence, prior inhibition of the task set for task A has occurred less recently, so there is less inhibition to overcome, resulting in relatively faster performance. Taskset inhibition is a robust phenomenon in task switching (Arbuthnott \& Frank, 2000; Hübner, Dreisbach, Haider, \& Kluwe, 2003; Mayr \& Keele, 2000; Schuch \& Koch, 2003) and is considered to be an executive control process that is distinct from negative priming (Mayr \& Keele, 2000), occurs for both perceptual (Mayr \& Keele, 2000) and semantic (Arbuthnott \& Frank, 2000) tasks, and may function to reduce task-set competition (Hübner et al., 2003), possibly by inhibiting category-response rules associated with irrelevant tasks (Schuch \& Koch, 2003).

Task-set inhibition is assumed to act on individual task sets, but the primary evidence for its existence comes from a sequential effect that is based on a contrast between different task sequences (ABA vs. CBA). In light of this fact, it may be important to move from the task level to the sequence level and consider how a task-level process, such as task-set inhibition, may be susceptible to hierarchical control from sequence-level representations and processes.

Schneider and Logan (2006) defined hierarchical control as a relationship between different levels of representation and processing, such that what happens at one level determines what happens at another level (see also Miller, Galanter, \& Pribram, 1960). Applied to task sequences, the sequence level can control the task level in two ways. First, a task sequence specifies whether and when different tasks must be performed, coordinating the serial order of task-level processes. Second, sequence-level processing may modulate task-level processing, because the processes

D.W. Schneider, darryl.schneider@vanderbilt.edu 
at each level act on representations in working memory. If sequence-level processing changes the contents of working memory, subsequent task-level processing could be altered.

Recent studies have provided evidence of hierarchical control between the sequence and task levels in taskswitching performance. For example, Lien and Ruthruff (2004) and Schneider and Logan (2006) found that when subjects repeated or switched between predictable task sequences, large switch costs within a sequence were eliminated or reversed between sequences (i.e., at sequence transitions). Schneider and Logan suggested that sequence-level processing associated with maintaining or instantiating a task sequence in working memory may perturb the relative activation of different task sets, thereby eliminating switch costs. An intriguing issue is whether this evidence of hierarchical control extends to task-set inhibition: Is the $n-2$ repetition cost affected by sequencelevel processing?

Task-set inhibition has been examined in the context of preplanned sequences (Mayr \& Keele, 2000, Experiment 5) and quasi-predictable sequences (Arbuthnott \& Frank, 2000), but only to ascertain the mere presence of $n-2$ repetition costs. However, a recent study by Koch, Philipp, and Gade (2006) represents the first attempt to explore the effects of sequence-level processing on taskset inhibition. Koch et al. were interested in the influence of task-sequence learning on task-set inhibition, and they conducted an experiment in which they examined $n-2$ repetition costs for the performance of a predictable sequence (of the form $\mathrm{ABACBC}$ ) and random sequences in subjects who were either instructed and aware or uninstructed and unaware of the predictable sequence. The critical finding was that for instructed-aware subjects, $n-2$ repetition cost was smaller with a predictable sequence than with random sequences; no such difference was present for uninstructedunaware subjects. Koch et al. hypothesized that for the instructed-aware subjects, "explicit instructions led to chunking of the task sequence, and that $n-2$ repetitions served as chunk points (ABA-CBC), so that within-chunk facilitation modulated the inhibition effect" (p. 346).

The hypothesis that within-chunk facilitation reduces task-set inhibition implies a form of hierarchical control, with chunking at the sequence level affecting task-set inhibition at the task level. However, Koch et al. (2006) noted that "future studies will have to corroborate our specific chunking hypothesis" (p. 350), for two important reasons. First, they had no direct evidence that their instructedaware subjects chunked the predictable sequence; chunking could only be inferred indirectly from the reduced $n-2$ repetition cost. Second, their use of a single predictable sequence $(\mathrm{ABACBC})$ did not allow them to evaluate the specific hypothesis of within-chunk facilitation and distinguish it from the more general hypothesis that any chunking of a sequence (with $n-2$ repetitions occurring within or between chunks) attenuates task-set inhibition.

\section{Testing the Chunking Hypothesis}

To extend Koch et al.'s (2006) work and provide additional evidence of a hierarchical control relationship between chunking and task-set inhibition, their chunk- ing hypothesis was tested in the following experiment. Subjects performed different task sequences in separate blocks of trials. In each block, subjects memorized a prescribed sequence, then performed the sequence repeatedly on a series of stimuli. No external task cues accompanied the stimuli (cf. Koch et al., 2006); therefore, subjects had to rely on their memories of the relevant sequence during the block.

There were two critical manipulations. First, subjects were induced to chunk each sequence into two groups of three tasks through spatial grouping (sequences were presented visually with a gap between the third and fourth tasks; for a related manipulation, see Lien \& Ruthruff, 2004) and instructions (chunking was explicitly recommended as a strategy to facilitate memorization of the sequences). Second, there were two types of sequences, one of the form ABACBC, and one of the form BACBCA. The first type is identical to that used by Koch et al. (2006), and it can be chunked such that both $n-2$ repetitions in the sequence occur within chunks (i.e., $\mathrm{ABA}-\mathrm{CBC}$, where the trials comprising sub-sequences of the form $\mathrm{ABA}$ occur in the same chunk). The second type is very similar to the first (only differing by a phase shift of one task), except that both $n-2$ repetitions in the sequence occur between chunks (i.e., BAC-BCA, where the trials comprising subsequences of the form ABA span two chunks).

There were two critical predictions. First, if subjects chunk each sequence, then there should be direct evidence of chunking in the form of chunk initiation effects. In response time (RT) data, chunk initiation effects reflect slower performance at the first serial position of a chunk in comparison with subsequent serial positions, and they are interpreted as clear evidence of chunking (Anderson, Bothell, Lebiere, \& Matessa, 1998; Koch \& Hoffmann, 2000; Logan, 2004; Povel \& Collard, 1982). Applied to the present study, mean RTs at the first and fourth serial positions of each sequence should be longer than mean RTs at the remaining serial positions. Second, if there is hierarchical control from the sequence level to the task level, such that within-chunk facilitation attenuates task-set inhibition, then $n-2$ repetition cost should be smaller when $n-2$ repetitions occur within chunks than between chunks. However, if any chunking of a sequence affects task-set inhibition, then comparable effects should be observed for $n-2$ repetitions occurring within and between chunks.

\section{METHOD}

\section{Subjects}

Thirty-two students from Vanderbilt University completed the experiment for partial course credit.

\footnotetext{
Apparatus, Stimuli, and Responses

The experiment was conducted using E-Prime software (Version 1.1; Psychology Software Tools, Pittsburgh, PA) operating on computers that displayed stimuli on monitors and registered responses from keyboards. The tasks were color, shape, and size judgments performed on a subset of eight stimuli created from the factorial combination of three dimensions: color (red or blue), shape (circle or triangle), and size $($ small $[3.5 \times 3.5 \mathrm{~cm}]$ or large $[7.0 \times 7.0 \mathrm{~cm}])$. All stimuli were displayed on a black background and viewed at a distance of about $60 \mathrm{~cm}$.
} 
Subjects responded to the stimuli by pressing the "Z" or "/" key with their left or right index fingers, respectively. Response categories for the same task were assigned to different response keys, and the eight possible response-key mappings were counterbalanced across subjects. Text indicating the response-key mappings appeared as a reminder in the bottom corners of the screen. For each subject, the two stimuli that were completely response congruent (i.e., stimuli that were associated with the same response key for all tasks) were excluded to prevent responding without performing the relevant task.

\section{Procedure}

Subjects were seated at computers in private testing rooms after providing informed consent. Instructions were presented onscreen and explained by the experimenter. There were 12 possible sequences - with 6 sequences of the form $\mathrm{ABA}-\mathrm{CBC}$ and 6 sequences of the form $\mathrm{BAC}-\mathrm{BCA}$ - that were based on all possible designations of the color, shape, and size tasks as A, B, or C for each type of sequence.

Subjects completed blocks of trials in which they were instructed to perform the tasks according to a sequence indicated at the start of each block, repeating the sequence every six trials. To encourage subjects to chunk each sequence, the relevant sequence for each block was displayed onscreen as a series of task names separated by commas, with a gap between the third and fourth task names to induce spatial grouping of the tasks into triplets. The experimenter highlighted this spatial grouping manipulation and explicitly recommended chunking as a strategy to facilitate memorization of the sequence.

Each block was preceded by a screen indicating the relevant sequence, which had to be memorized because no external task cues or any reminder about the sequence were presented during the block. Subjects completed two practice blocks - one block for each type of sequence ( $\mathrm{ABA}-\mathrm{CBC}$ or $\mathrm{BAC}-\mathrm{BCA})$, with the task-specific sequence randomly selected. Subjects then completed 12 experimental blocks - 1 block for each possible task-specific sequence, randomly selected, with the restriction that each type of sequence be used once every two blocks. Blocks were separated by rest periods.

There were 36 trials per block, allowing six iterations of the sequence and six presentations of each stimulus. Each trial began with the onset of a randomly selected stimulus in the center of the screen, which remained onscreen until the subject responded. The stimulus was then erased, and the next trial commenced after a response-stimulus interval of $200 \mathrm{msec}$. Note that there was no spatial or temporal grouping of stimuli. The RT and response were recorded for each trial. After the experiment, which lasted $25 \mathrm{~min}$, subjects were debriefed and thanked for their participation.

\section{RESULTS}

Practice blocks, the first three trials of each block, and trials with an RT exceeding 4,000 msec (1.7\% of trials) were excluded from all analyses. Blocks with error rates exceeding $15 \%$ were also excluded $(M=0.59$ blocks per subject). A block error-rate criterion was implemented to account for those rare instances when subjects forgot the sequence during the block. Mean error rate was $2.4 \%$, and the error data were consistent with the RT data (except where noted); therefore, the analyses focused on the latter. Only correct trials that were preceded by at least two correct trials were included in the RT analyses.

\section{Chunk Initiation Effects}

To identify chunk initiation effects, the mean RT was calculated for each serial position in each sequence (see Figure 1). Chunk initiation effects are clearly evident for both sequences, an observation supported by separate one- way repeated-measures ANOVAs with serial position as the factor. The effect of serial position was significant for both sequences $\left[\mathrm{ABA}-\mathrm{CBC}, F(5,155)=43.71, M S_{\mathrm{e}}=\right.$ $17,231.97, p<.001, \eta_{\mathrm{p}}^{2}=.59 ; \mathrm{BAC}-\mathrm{BCA}, F(5,155)=$ 51.81, $\left.M S_{\mathrm{e}}=27,321.92, p<.001, \eta_{\mathrm{p}}^{2}=.63\right]$. Planned contrasts were conducted to test specifically for chunk initiation effects, using weights of $+2,-1,-1,+2,-1$, and -1 (for Serial Positions 1-6, respectively) to see whether the mean RTs for the first and fourth serial positions were longer than the mean RTs for the remaining serial positions (ignoring task-set inhibition effects, which are analyzed below). The contrast was significant for both sequences [ABA-CBC, $F(1,155)=186.23, p<.001$, $\eta_{\mathrm{p}}^{2}=.55 ; \mathrm{BAC}-\mathrm{BCA}, F(1,155)=161.73, p<.001, \eta_{\mathrm{p}}^{2}=$ $.51]$. These results indicate that subjects reliably chunked the sequences into triplets, providing justification for analyses of task-set inhibition effects occurring within and between chunks. ${ }^{1}$

\section{Task-Set Inhibition Effects}

In order to investigate putative task-set inhibition effects, two types of analyses were conducted: sequence and serial position analyses. Both analyses involve a contrast between $n-2$ repetition costs calculated from $n-2$ repetitions occurring within versus between chunks, but they make different assumptions and control for different factors that may affect performance.

For the sequence analysis, $n-2$ repetition costs were calculated from $n-2$ repetitions and switches in the same sequence (similar to the analysis of Koch et al., 2006). This analysis controls for potential differences across sequences (which have been shown to affect overall RT; see Schneider \& Logan, 2006, Experiment 2), but assumes that chunk initiation effects are restricted to the first serial position of each chunk (consistent with the data of Logan, 2004, and Schneider \& Logan, 2006) and that it does not matter if $n-2$ switches occur within or between chunks (these assumptions are addressed in the serial position analysis).

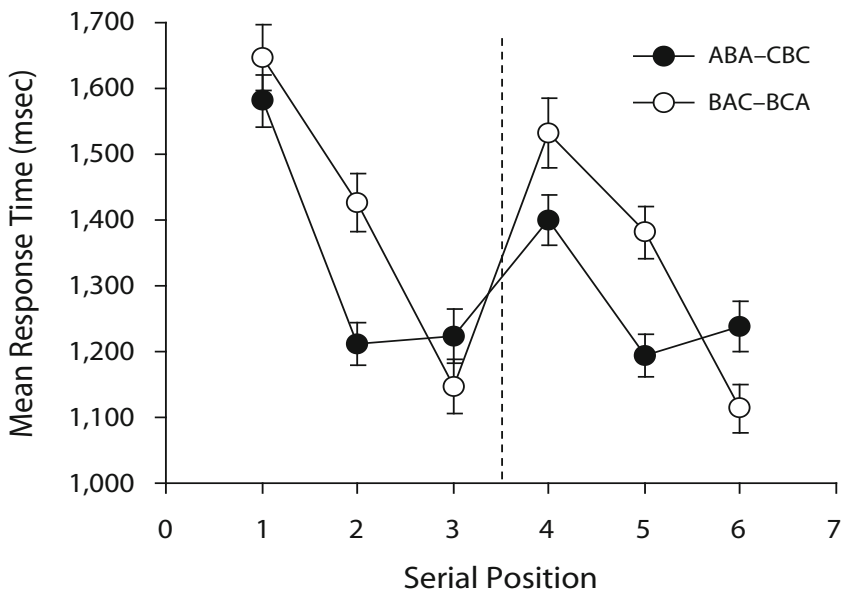

Figure 1. Mean response time as a function of serial position and sequence ( $\mathrm{BBA}-\mathrm{CBC}$ or $\mathrm{BAC}-\mathrm{BCA})$. The dashed vertical line represents a hypothesized chunk boundary. Error bars represent standard errors of the means. 
For each chunk in the sequences, $n-2$ repetition costs were calculated as follows: For the ABA-CBC sequence, the mean RT for the second serial position of the chunk (an $n-2$ switch occurring between chunks) was subtracted from the mean RT for the third serial position of the chunk (an $n-2$ repetition occurring within chunks). For the BAC-BCA sequence, the mean RT for the third serial position of the chunk (an $n-2$ switch occurring within chunks) was subtracted from the mean RT for the second serial position of the chunk (an $n-2$ repetition occurring between chunks). These calculations are equivalent to taking the differences between points on the same line in Figure 1. The mean RT for the first serial position of each chunk was not included in the calculations, because the chunk initiation effects associated with the first serial position would alter the $n-2$ repetition costs for reasons unrelated to task-set inhibition.

If within-chunk facilitation attenuates task-set inhibition, then the $n-2$ repetition cost should be smaller for $n-2$ repetitions occurring within chunks than between chunks. A 2 (chunk: first or second) $\times 2$ ( $n-2$ repetition locus: within or between chunks) ANOVA on the $n-2$ repetition costs revealed a significant main effect of locus $\left[F(1,31)=34.15, M S_{\mathrm{e}}=56,242.53, p<.001, \eta_{\mathrm{p}}^{2}=.52\right]$. The $n-2$ repetition cost was smaller for $n-2$ repetitions occurring within chunks $(M=29 \mathrm{msec})$ than between chunks $(M=274 \mathrm{msec}$; see Figure 2), supporting the chunking hypothesis. The main effect of chunk and the chunk $\times$ locus interaction were not significant $[F \mathbf{s}<1]$.

For the serial position analysis, $n-2$ repetition costs were calculated from $n-2$ repetitions and switches at the same serial position. This analysis complements the sequence analysis, because it assumes that chunk initiation effects are not necessarily restricted to the first serial position of each chunk (i.e., there may be a carryover effect to subsequent serial positions), and it controls for whether $n-2$ switches occur within or between chunks, but assumes that there are no differences across sequences.

For each chunk in the sequences, $n-2$ repetition costs were calculated as follows: For transitions occurring between chunks, the mean RT for the second serial position of the chunk in the ABA-CBC sequence (an $n-2$ switch occurring between chunks) was subtracted from the mean RT for the second serial position of the chunk in the BAC-BCA sequence (an $n-2$ repetition occurring between chunks). For transitions occurring within chunks, the mean RT for the third serial position of the chunk in the BAC-BCA sequence (an $n-2$ switch occurring within chunks) was subtracted from the mean RT for the third serial position of the chunk in the ABA-CBC sequence (an $n-2$ repetition occurring within chunks). These calculations are equivalent to taking the differences between points at the same serial position, but on different lines in Figure 1. Note that these calculations involve the same data as those in the sequence analysis; $n-2$ repetition costs are just calculated from different groupings of the same means.

If within-chunk facilitation attenuates task-set inhibition, then $n-2$ repetition cost should be smaller for $n-2$ repetitions occurring within chunks than between chunks. A 2 (chunk: first or second) $\times 2(n-2$ repetition locus: within or between chunks) ANOVA on the $n-2$ repetition costs revealed a significant main effect of locus $\left[F(1,31)=6.57, M S_{\mathrm{e}}=50,056.83, p<.05, \eta_{\mathrm{p}}^{2}=.17\right]$. The $n-2$ repetition cost was smaller for $n-2$ repetitions occurring within chunks $(M=101 \mathrm{msec})$ than between chunks ( $M=202 \mathrm{msec}$; see Figure 2), consistent with the sequence analysis. ${ }^{2}$ The main effect of chunk was not significant $[F<1]$, nor was the chunk $\times$ locus interaction $\left[F(1,31)=2.59, M S_{\mathrm{e}}=16,646.93, p=.12\right]$.

\section{DISCUSSION}

Hierarchical control in the performance of task sequences was investigated by examining how chunking (a sequence-level process) affects task-set inhibition (a task-level process). An experiment that was conducted to test Koch et al.'s (2006) hypothesis that within-chunk facilitation reduces task-set inhibition provided direct evidence of chunking (in the form of chunk initiation effects), and the sequence and serial position analyses of task-set inhibition effects both indicated that differences in chunking affected performance: The $n-2$ repetition cost was smaller when $n-2$ repetitions occurred within chunks than between chunks. These results complement Koch et al.'s findings and are suggestive of hierarchical control, with chunking affecting task-set inhibition.

\section{Elaborating the Chunking Hypothesis}

Although Koch et al. (2006) hypothesized that the reduction in $n-2$ repetition cost in their experiment was due to within-chunk facilitation, they suggested that instead of modulating task-set inhibition per se, chunking may facilitate performance by priming task goals, "particularly those goals that refer to the $n-2$ task repetitions, which

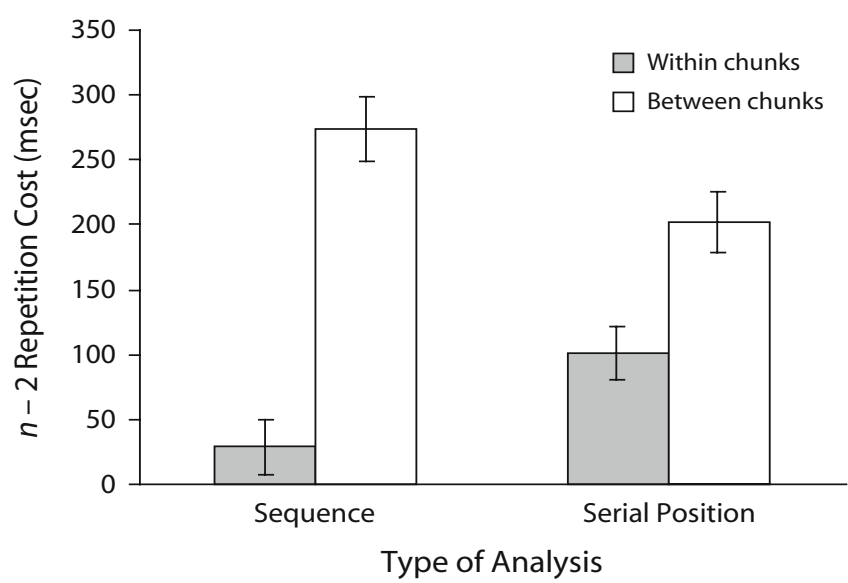

Figure 2. Mean $n-2$ repetition cost as a function of type of analysis (sequence or serial position) and $n-2$ repetition locus (within or between chunks). Error bars represent standard errors of the means. 
are located within a chunk" (p. 349). This suggestion is consistent with an elaborated version of Koch et al.'s hypothesis that is based on two main assumptions.

The first assumption is that only one chunk can be loaded into working memory at a time. This assumption is supported empirically by the presence of a chunk initiation effect for the second chunk of each sequence in the present experiment (see Figure 1), which suggests that subjects loaded individual chunks rather than the entire sequence into working memory.

The second assumption is that a meaningful distinction can be made between a task goal (i.e., what task has to be performed) and a task set (i.e., how a task is performed). The former is arguably a prerequisite for the latter, in that one must instantiate a task goal in working memory to enable selection of an appropriate task set, similar to how a relevant goal must be available in working memory to enable firing of condition-action rules in production system models of cognition (see, e.g., Anderson, 1993). Loading a chunk of a task sequence into working memory can be construed as instantiating a serially ordered list of task goals in working memory (Schneider \& Logan, 2006) for subsequent selection of task sets. If one defines a task set as a set of rules for mapping stimulus categories onto responses, then this distinction between goals and task sets is consistent with the separate stages of goal shifting and rule activation in the task-switching model of Rubinstein, Meyer, and Evans (2001). Furthermore, if one endorses the proposal that task-set inhibition involves inhibiting category-response rules associated with irrelevant tasks
(Schuch \& Koch, 2003), then one can argue that task-set inhibition acts on task sets rather than on task goals.

Given these assumptions, the pattern of data in the present experiment can be explained as a combination of the priming of task goals and task-set inhibition, using Rubinstein et al.'s (2001) model as a conceptual framework. Goal shifting and rule activation stages for $n-2$ switches and repetitions are depicted in Figure 3 for $\mathrm{BAC}-\mathrm{BCA}$ and $\mathrm{ABA}-\mathrm{CBC}$ sequences. The priming of task goals is assumed to facilitate goal shifting, shortening the duration of the goal shifting stage. Task-set inhibition is assumed to impair rule activation for $n-2$ repetitions, prolonging the duration of the rule activation stage. In tandem, goal activation and task-set inhibition can yield the observed pattern of data in the following manner.

When an $n-2$ repetition occurs between chunks - as in a BAC-BCA sequence-subjects will not know that the task at the third serial position of the first chunk will be repeated at the second serial position of the second chunk if only the first chunk is available in working memory. Subjects may recognize that $n-2$ repetitions occur across chunks in BAC-BCA sequences, but if trial-to-trial performance is based on a single chunk in working memory, then they may be unable to prime the task goals in the next chunk. Consequently, the task goal associated with the $n-2$ repetition would not be primed and goal shifting would not be facilitated, resulting in no offset of the cost associated with task-set inhibition. The net effect would be a substantial $n-2$ repetition cost (see Figure 3).

BAC-BCA sequence:

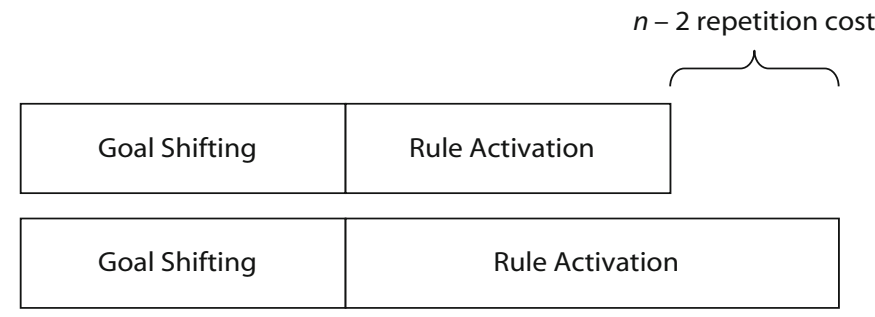

$A B A-C B C$ sequence:

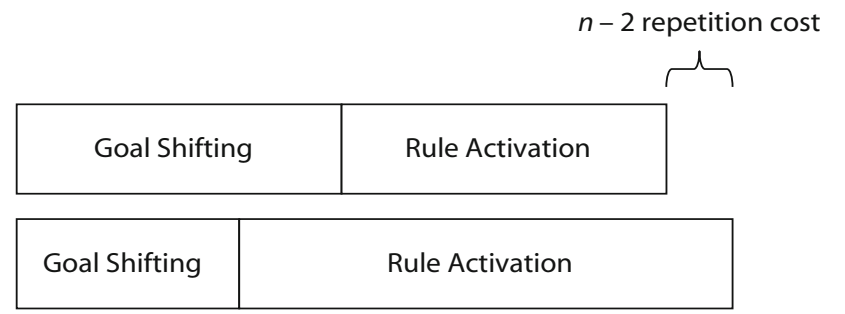

Figure 3. Schematic depiction of how the reduced $n-2$ repetition cost associated with $n-2$ repetitions occurring within chunks can be interpreted as a net effect of activation and inhibition affecting separate stages of goal shifting and rule activation, respectively, in the context of Rubinstein et al.'s (2001) model of task switching (with the length of the box associated with each stage reflecting its duration, and additional processing stages omitted for simplicity). Task-set inhibition is assumed to impair rule activation for $n-2$ repetitions, prolonging the duration of the rule activation stage. For $n-2$ repetitions occurring between chunks, this yields a sizable $n-2$ repetition cost, as depicted for the $\mathrm{BAC}-\mathrm{BCA}$ sequence. Chunking is assumed to facilitate performance of $\boldsymbol{n - 2}$ repetitions occurring within chunks by priming task goals, shortening the duration of the goal shifting stage. The net effect of goal activation and task-set inhibition is a reduced $n-2$ repetition cost, as depicted for the ABA-CBC sequence. Note that the manner in which $n-2$ repetition costs are depicted corresponds to the sequence analysis. 
When an $n-2$ repetition occurs within chunks - as in an ABA-CBC sequence-subjects will know that the task at the first serial position will be repeated at the third serial position as soon as the relevant chunk is loaded into working memory. This foreknowledge could prime the task goal associated with the $n-2$ repetition, facilitating subsequent goal shifting and partially offsetting the cost associated with task-set inhibition. The net effect would be a reduced $n-2$ repetition cost (see Figure 3 ), but without direct modulation of task-set inhibition.

Consequently, the elaborated hypothesis provides a tenable account of the present data by construing the $n-2$ repetition cost as a net effect of goal activation and task-set inhibition, leading to two important implications. First, $n-2$ repetition costs may not be pure measures of task-set inhibition. Second, instead of chunking modulating task-set inhibition, hierarchical control may be better characterized as a functional relationship between different types of processing (Schneider \& Logan, 2006). ${ }^{3}$ Rather than diminishing the meaning of hierarchical control, this characterization provides novel insight into how such control might be manifest in behavioral measures of task-switching performance.

\section{Contrasting Effects of Hierarchical Control}

In the present study, hierarchical control was inferred from a smaller $n-2$ repetition cost for $n-2$ repetitions occurring within chunks than between chunks. In other studies, hierarchical control was inferred from eliminated or reversed $n-1$ switch costs (differences between immediate task switches and task repetitions) for transitions occurring between chunks than within chunks (or sequences; Lien \& Ruthruff, 2004; Schneider \& Logan, 2006). ${ }^{4}$ These contrasting effects cannot be explained by pure activation- or inhibition-based accounts of task-switching performance, suggesting that hybrid models of task-set control involving both activation and inhibition may be required to explain the intricacies of hierarchical control. Indeed, the elaborated hypothesis outlined in this article is based on the idea that activation and inhibition both contribute to $n-2$ repetition costs occurring in chunked task sequences. Future studies aimed at investigating the relationship between these contrasting effects - augmented by attempts to model them within a common framework - may yield better insight into how the mind controls itself.

\section{AUTHOR NOTE}

The author thanks Gordon Logan for use of his laboratory facilities (funded by Grant BCS 0446806 from the National Science Foundation) and Katherine Arbuthnott, Iring Koch, Gordon Logan, Nachshon Meiran, Tram Neill, and Eric Ruthruff for helpful comments on this work. Correspondence concerning this article should be addressed to D. W. Schneider, Department of Psychology, Vanderbilt University, Nashville, Tennessee 37203 (e-mail: darryl.schneider@vanderbilt.edu).

\section{REFERENCES}

Anderson, J. R. (1993). Rules of the mind. Hillsdale, NJ: Erlbaum. Anderson, J. R., Bothell, D., Lebiere, C., \& Matessa, M. (1998). An integrated theory of list memory. Journal of Memory \& Language, 38, 341-380.

Arbuthnott, K., \& Frank, J. (2000). Executive control in set switch- ing: Residual switch cost and task-set inhibition. Canadian Journal of Experimental Psychology, 54, 33-41.

Hübner, M., Dreisbach, G., Haider, H., \& Kluwe, R. H. (2003). Backward inhibition as a means of sequential task-set control: Evidence for reduction of task competition. Journal of Experimental Psychology: Learning, Memory, \& Cognition, 29, 289-297.

Koch, I., \& Hoffmann, J. (2000). Patterns, chunks, and hierarchies in serial reaction-time tasks. Psychological Research, 63, 22-35.

Koch, I., Philipp, A. M., \& Gade, M. (2006). Chunking in task sequences modulates task inhibition. Psychological Science, 17, 346350 .

Lien, M.-C., \& Ruthruff, E. (2004). Task switching in a hierarchical task structure: Evidence for the fragility of the task repetition benefit. Journal of Experimental Psychology: Learning, Memory, \& Cognition, 30, 697-713.

LoGAN, G. D. (2004). Working memory, task switching, and executive control in the task span procedure. Journal of Experimental Psychology: General, 133, 218-236.

Mayr, U., \& Keele, S. W. (2000). Changing internal constraints on action: The role of backward inhibition. Journal of Experimental Psychology: General, 129, 4-26.

Miller, G. A., Galanter, E., \& Pribram, K. H. (1960). Plans and the structure of behavior. New York: Holt, Rinehart \& Winston.

Monsell, S. (2003). Task switching. Trends in Cognitive Sciences, 7 , 134-140.

Povel, D.-J., \& Collard, R. (1982). Structural factors in patterned finger tapping. Acta Psychologica, 52, 107-123.

Rubinstein, J. S., Meyer, D. E., \& Evans, J. E. (2001). Executive control of cognitive processes in task switching. Journal of Experimental Psychology: Human Perception \& Performance, 27, 763-797.

Schneider, D. W., \& Logan, G. D. (2006). Hierarchical control of cognitive processes: Switching tasks in sequences. Journal of Experimental Psychology: General, 135, 623-640.

ScHUCH, S., \& KocH, I. (2003). The role of response selection for inhibition of task sets in task shifting. Journal of Experimental Psychology: Human Perception \& Performance, 29, 92-105.

\section{NOTES}

1. Comparable analyses of the error data revealed only one result that was discrepant with the RT data. A one-way ANOVA on the ABA-CBC sequence data - with serial position as the factor-yielded a significant effect of serial position $\left[F(5,155)=2.54, M S_{\mathrm{e}}=7.96, p<.05, \eta_{\mathrm{p}}^{2}=\right.$ .08]. The same contrast that was used to test for chunk initiation effects was significant $\left[F(1,155)=3.92, p<.05, \eta_{\mathrm{p}}^{2}=.02\right]$, but it reflected lower error rates for the first and fourth serial positions $(M=2.1 \%)$ than for the remaining serial positions $(M=2.9 \%)$, suggesting a possible speed-accuracy trade-off. To examine this issue in greater detail, mean RTs were calculated separately for error and correct responses for the ABA-CBC sequence, collapsing across serial position (because $51 \%$ of the cells associated with error responses were empty due to the low error rates) and excluding one subject who did not make any errors. If there was a speed-accuracy trade-off, then error responses should have been significantly faster than correct responses. However, the difference between response types was not significant $[F(1,30)=1.28$, $\left.M S_{\mathrm{e}}=85,343.61, p=.27\right]$, and it was in the opposite direction, with numerically longer RTs for error responses $(M=1,411 \mathrm{msec})$ compared with correct responses $(M=1,327 \mathrm{msec})$. These results, coupled with the lack of evidence for a speed-accuracy trade-off in the BAC-BCA sequence data [a one-way ANOVA on the error data did not reveal a significant effect of serial position, $F(5,155)=1.09, M S_{\mathrm{e}}=8.48, p=$ .37], indicate that the RT data are not compromised.

2. Although the sequence and serial position analyses agreed with each other, they could have yielded discrepant results if an assumption underlying either analysis was violated. For example, imagine that there was no difference in the $n-2$ repetition cost as a function of $n-2$ repetition locus, but overall RT differed across sequences (with BAC-BCA > ABA-CBC), violating an assumption of the serial position analysis. With a serial position analysis, one would find that $n-2$ repetition cost is smaller for $n-2$ repetitions occurring within chunks than between chunks, but that would be an artifact arising from the difference in overall RT across sequences. However, with a sequence analysis, one would find no difference in $n-2$ repetition cost because that analysis controls 
for differences in overall RT across sequences. This example illustrates the importance of noting the extent to which the analyses agree with each other, because that reflects the degree to which their underlying assumptions are valid. The differences in absolute $n-2$ repetition costs across analyses (see Figure 2) suggest a possible minor violation of an assumption, but the critical finding is that both analyses yielded the same relative pattern of $n-2$ repetition costs.

3. To be more precise, if one holds Rubinstein et al.'s (2001) view that goal shifting and rule activation are executive control processes, and Mayr and Keele's (2000) view that task-set inhibition is an executive control process, then hierarchical control can be interpreted as a sequence-level process (chunking) modulating an executive control process (goal shifting) to offset the effect of another executive control process (task-set inhibition) acting on yet another executive control process (rule activation).

4. The author thanks Eric Ruthruff for highlighting these contrasting effects.

(Manuscript received June 23, 2006; revision accepted for publication September 30, 2006.) 